\title{
Funciones ejecutivas en niños escolarizados con alta y baja creatividad
}

\section{Executive functions in School-aged Children with high and low Creativity}

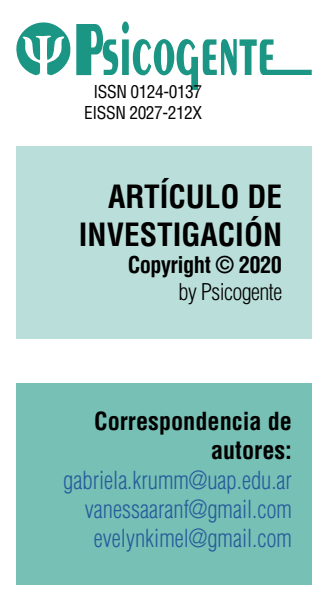

Recibido: $15-07-2019$ Aceptado: $31-03-2020$

Publicado: 05-06-20

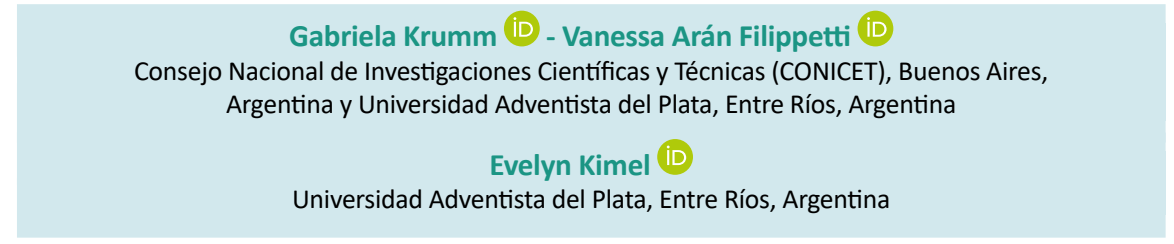

Resumen

Objetivo: El presente estudio tuvo como objetivo analizar el perfil de funcionamiento ejecutivo característico en niños creativos.

Método: Participaron 200 niños y niñas escolarizados de la provincia de Entre Ríos, República Argentina seleccionados por medio de un muestreo intencional. La creatividad se evaluó por medio de la prueba de figuras del Test de Pensamiento Creativo de Torrance (TTCT), Forma A y la prueba verbal CREA C, inteligencia creativa. Como medida de las Funciones Ejecutivas (FE), se emplearon diferentes pruebas para valorar cada dominio: (1) subtest de memoria de trabajo del WISC IV; (2) Stroop, test de colores y palabras, para inhibición; (3) WCST computarizado, para flexibilidad cognitiva reactiva y Fluidez verbal y Five Point Test para fluidez cognitiva espontánea, y (4) Laberintos de Porteus para valorar la planificación. Para estudiar perfiles de funcionamiento ejecutivo se realizaron MANCOVAS ingresando la inteligencia como covariable.

Resultados: Los resultados del presente estudio sugieren la existencia de un perfil de FE en niños creativos en función de su desempeño en la prueba de figuras del TTCT (F de Hotelling $(7,90)=3.404$; $p=.003, \eta^{2}$ parcial $=.21$ ) y del CREA C (F de Hotelling $(7,98)=8.831 ; p<.001, \eta^{2}$ parcial $\left.=.39\right)$.

Conclusión: Los hallazgos del presente estudio muestran que los niños con mayor creatividad, tanto en tareas de dibujo como en tareas verbales, presentan un perfil de FE caracterizado por una mayor capacidad de memoria de trabajo, inhibición y flexibilidad cognitiva espontánea. Sin embargo, parecería que la naturaleza de la tarea es un factor que podría modular la contribución de los procesos cognitivos de alto orden al potencial creativo.

Palabras clave: procesos cognitivos, pensamiento divergente, creatividad, funciones ejecutivas, inhibición, flexibilidad cognitiva, memoria de trabajo, planificación, niños escolarizados.

\section{Abstract}

Objective: The objective of this study is to analyze the executive functioning profile, characteristic in creative children.

Method: The participants were 200 school children from the province of Entre Ríos, Argentina, selected through intentional sampling. Creativity was assessed using the figure test of Torrance Tests of Creative Thinking (TTCT), Form A and the CREA C verbal test, creative intelligence. To measure executive functions (EF), different tests were used to assess each domain: (1) WISC IV Working Memory Subtest; (2) Stroop Color and Word Test for inhibition; (3) computerized WCST for reactive cognitive flexibility and verbal fluency, the Five Point Test for spontaneous cognitive fluency, and finally; (4) Porteus Maze test to assess planning. Multivariate analysis of covariance (MANCOVAS) was performed to study executive functioning profiles, entering intelligence as a covariate. 
Results: The study results suggest the existence of an EF profile in creative children based on their performance in the TTCT figure test (Hotelling's $F(7,90)=3.404, p=.003, \eta^{2}$ partial $=0.21$ ) and from CREA C (Hotelling's $F(7,98)=8.831 ; p<0.001, \eta^{2}$ partial $=0.39$ ).

Conclusions: The findings of this study show that children with greater creativity, both in drawing and verbal tasks, show an EF profile characterized by a greater capacity for working memory, inhibition, and spontaneous cognitive flexibility. However, due to the task's nature, the high-order cognitive processes could contribute in a greater or lesser extent to creative potential.

Keywords: cognitive processes, divergent thinking, creativity, executive functions, inhibition, cognitive flexibility, working memory, planning, schooled children.

\section{INTRODUCCIÓN}

En los últimos años se ha evidenciado un progresivo interés por el estudio de la correspondencia entre la creatividad y las Funciones Ejecutivas (en adelante, FE). La evidencia empírica muestra que las FE (Gilhooly, Fioratou, Anthony \& Wynn, 2007; Nusbaum \& Silvia, 2011) cumplen un rol importante en la creatividad, abriendo nuevas líneas de investigación para examinar cómo los mecanismos cognitivos de orden superior pueden contribuir al pensamiento creativo. Sin embargo, los estudios, en general, se han enfocado en poblaciones adultas, no dejando claro cómo es manifiesta esta relación en los niños.

La creatividad ha sido definida como la más alta expresión de las nuevas ideas, de la flexibilidad de perspectivas, del cambio, de la capacidad de combinar conceptos no relacionados de diferentes formas y de evitar los caminos comunes (Benedek, Franz, Heene \& Neubauer, 2012; Benedek, Könen \& Neubauer, 2012; Chi, 1997). Esta definición sugiere que el pensamiento creativo requiere de las $\mathrm{FE}$, procesos cognitivos de alto orden que posibilitan el control de la actividad cognitiva, conductual y emocional. Se trata de constructo multidimensional que engloba los subprocesos de memoria de trabajo, inhibición y flexibilidad cognitiva (Diamond, 2016; Friedman et al., 2006; Miyake et al., 2000). La planificación, además, se considera una FE más avanzada que se construye a partir de los tres componentes ejecutivos centrales (Diamond, 2016; Miyake et al., 2000). Estas habilidades se asocian a la actividad de la corteza prefrontal (CPF) y conexiones recíprocas con otras áreas corticales y estructuras subcorticales (Fuster, 1997; Heyder, Suchan \& Daum, 2004). Consistentemente, recientes avances en neurociencia han demostrado que el lóbulo frontal, así como regiones posteriores del cerebro y estructuras subcorticales (Dietrich, 2004; Heilman, Nadeau \& Beversdorf, 2003) se ven implicados en el proceso creativo. Tanto la creatividad (ver Gajda, Karwowski \& Beghetto, 2017, para una revisión) como las FE (Arán Filippetti \& Richaud, 2015, 2017) son constructos relacionados con rendimiento académico en edad escolar. 
Diferentes estudios han analizado la relación entre la creatividad y los diversos componentes del constructo FE en poblaciones adultas. Las investigaciones muestran correlaciones positivas entre la memoria de trabajo y el rendimiento creativo en diferentes tareas (De Dreu, Nijstad, Baas, Wolsink \& Roskes, 2012; Oberauer, Süß Wilhelm, \& Wittmann, 2008; Sub, Oberauer, Wittmann, Wilhelm \& Schulze, 2002). Se ha sugerido que la memoria de trabajo cumpliría dos funciones básicas: mantener la información novedosa en un estado elevado de actividad y discriminar entre información relevante e irrelevante para la tarea (Unsworth \& Engle, 2007). Estas dos funciones son importantes para la creatividad, ya que la misma requiere la superación de las tendencias habituales tales como, la generación de las ideas accesibles pero comunes y la consideracion de las perspectivas y puntos de vista iniciales. Además, el pensamiento creativo requiere la combinación de conceptos recuperados de la memoria a largo plazo para construir nuevas ideas y soluciones de problemas (Nijstad \& Stroebe, 2006 citado en De Dreu et al., 2012). En la misma línea, Lee \& Therriault (2013) mencionan que la memoria de trabajo cumple un papel importante en la creatividad, ya que los individuos con alta capacidad de memoria de trabajo tienen más probabilidades de superar la interferencia causada por respuestas automáticas y no originales, siendo más exitosos en el uso de estrategias para generar nuevos enfoques y respuestas en tareas de pensamiento creativo.

En cuanto a inhibición, la literatura muestra evidencias sobre su asociación con el pensamiento divergente (Golden, 1975). En forma específica con la fluidez ideacional, es decir, con la capacidad de producir una serie de ideas en forma rápida (Benedek, Franz et al., 2012), y con la flexibilidad, pero no con originalidad en tareas de resolución de problemas (Cheng, Hu, Jia \& Runco, 2016). Estudios en esta línea han sugerido que la creatividad está vinculada con una mejor inhibición de la respuesta (Edl, Benedek, Papousek, Weiss \& Fink, 2014; Groborz \& Necka, 2003), así como con una capacidad elevada para evitar respuestas repetitivas (Benedek, Franz et al., 2012; Zabelina, Robinson, Council \& Bresin, 2012). Por otro lado, también se ha encontrado que las personas creativas parecen mostrar un pensamiento exclusivo y una disminución del filtrado de información irrelevante para la tarea (Carson, Peterson \& Higgins, 2003). La evidencia arrojada por estudios previos muestra que la falta de control cognitivo beneficia la creatividad (Martindale, 1999; Thompson-Schill, Ramscar \& Chrysikou, 2009) y que los sujetos creativos pueden enfocar o desenfocar la atención en función de las demandas de la tarea. Si bien existe evidencia de que la creatividad está relacionada con la inhibición cognitiva, la misma no es concluyente (Benedek, Franz et al., 2012). 
Respecto de la relación entre la creatividad y la flexibilidad cognitiva las teorías sobre creatividad subrayan la importancia de la flexibilidad de pensamiento para evitar los caminos comunes, considerando y combinando múltiples conceptos (Benedek, Jauk, Sommer, Arendasy \& Neubauer, 2014). En efecto, el dual pathway to creativity model (DPCM; De Dreu, Baas \& Nijstad, 2008) postula a la flexibilidad cognitiva como un aspecto crucial, al proponer que el desempeño creativo depende de (1) persistencia y de (2) flexibilidad. En este sentido, la relación entre flexibilidad cognitiva y creatividad se ve respaldada por el hecho de que la creatividad requiere de flexibilidad de pensamiento (Chi, 1997) para realizar saltos mentales entre diferentes categorías y producir ideas novedosas (Pan \& Yu, 2018). Consistentemente, estudios previos han mostrado correlaciones positivas entre la creatividad y la fluidez y flexibilidad asociativa (Benedek, Könen et al., 2012) y que la fluidez ejecutiva predice la producción en tareas de usos nuevos, mientras que no es predictor de los usos familiares (Gilhooly et al., 2007).

Finalmente, la investigación sobre la relación entre la planificación y la creatividad es muy escasa y se ha llevado a cabo principalmente con poblaciones de jóvenes adultos. Algunos estudios en esta línea han demostrado que el entrenamiento en habilidades de planificación, como la previsión y la identificación de causas, se relacionan con un mejor desempeño en tareas de pensamiento divergente (Osburn \& Mumford, 2006) y tareas creativas de resolución de problemas (Marta, Leritz \& Mumford, 2005).

Si bien existe evidencia empírica sobre la relación entre la creatividad y las FE en adultos, la misma se ha examinado en forma parcial, reportándose vínculos entre la creatividad y memoria de trabajo, creatividad y flexibilidad y creatividad e inhibición; sin tener en cuenta la compleja relación entre todos los componentes del constructo FE y la creatividad verbal vs figuras. Además, los estudios en general se han realizado en adultos, no siendo claro el papel que tienen las FE en la creatividad en los niños y, en particular, el rol de la planificación. Por otro lado, dado que tanto la creatividad como las FE se encuentran en pleno desarrollo durante la niñez (Arán Filippetti, 2013; De Luca et al., 2003; Huizinga, Dolan \& Van Der Molen, 2006; Krumm, Arán Filippetti, Lemos, Aranguren \& Vargas Rubilar, 2013), las relaciones y configuraciones podrían ser diferentes a las observadas en adultos. Por último, considerando que diferentes estudios muestran que la creatividad y las FE se relacionan con el rendimiento académico (Arán Filippetti \& Richaud, 2015, 2017; Gajda, Karwowski \& Beghetto, 2017; Mourgues, Tan, Hein, Elliott \& Grigorenko, 2016; ver también Ávila, López-Fernández \& Arias-Castro, 2018), 
el estudio de estos constructos tiene importantes implicaciones para el currículo escolar. En función de lo expuesto, el objetivo del presente trabajo es estudiar el perfil de FE (memoria de trabajo, inhibición, flexibilidad cognitiva y planificación) en función de dos medidas para evaluar la creatividad desde el pensamiento divergente, una verbal y la otra de dibujos o figuras (i.e., CREA vs TTCT figuras), en niños escolarizados de 8 a 13 años de edad.

\section{MÉTODO}

\subsection{Diseño y participantes}

El estudio corresponde a un diseño de diferencias de grupos, de corte transversal. La muestra, obtenida a través de un muestreo no probabilístico por conveniencia, estuvo conformada por 200 niños escolarizados hispanohablantes residentes en Argentina, de los cuales 86 (43\%) eran varones y 114 (57 \%) eran mujeres. La media de edad fue de 10.01, siendo el desvío estándar de 1.24. Los alumnos evaluados pertenecían a diferentes niveles educativos, que iban desde el tercer grado de la escuela primaria al primer año de la escuela secundaria de diferentes zonas de la provincia de Entre Ríos, Argentina. Los criterios de inclusión que se tuvieron en cuenta fueron: (a) niños que no presenten antecedentes clínicos, neurológicos ni psiquiátricos, (b) que cursen estudios escolares con regularidad, (c) sin repitencia escolar. Además, se evaluó la inteligencia como variable de control.

\subsection{Instrumentos}

\subsubsection{La prueba de Figuras del Test de Pensamiento Creativo de Torrance} (TTCT, Torrance, Ball \& Safter, 1992), Forma A

La prueba de Figuras del TTCT está constituida por tres actividades de 10 minutos cada una. Las mismas evalúan las habilidades: Fluidez, Originalidad, Elaboración, Resistencia al Cierre Prematuro y Abstracción de título (Torrance et al., 1992). En cuanto a la confiabilidad, el estudio en niños y adolescentes argentinos de la prueba de Figuras en su Forma B, presentó una consistencia interna .70 (Krumm \& Lemos, 2011). En relación con la validez de constructo, el Análisis Factorial Confirmatorio con la Forma A identificó dos factores correlacionados, Innovación y Adaptación, el primer factor compuesto por las habilidades fluidez y originalidad, y el segundo por las habilidades elaboración, resistencia al cierre prematuro y abstracción de títulos (Krumm, Arán Filippetti, Lemos, Koval \& Balabanian, 2016). El test permite obtener una puntuación total, que es la que se utilizó en este trabajo. 


\subsubsection{CREA, Inteligencia Creativa}

La prueba consta de tres láminas estímulo ( $\mathrm{A}$, B y C, dependiendo las edades) a partir de las cuales los entrevistados deben formular la mayor cantidad de interrogantes posibles en un tiempo establecido (4 minutos), después de que se dan las instrucciones especificadas en el manual del CREA. La prueba es de aplicación individual o colectiva y se puede utilizar desde los 6 años de edad. En este trabajo de utilizó el CREA C para niños y adolescentes, siendo la aplicación en niños de 8 y 9 años individual y en los demás colectiva. El estudio de confiabilidad entre las formas A y B, como formas paralelas, fue de 87. En relación con la validez, los autores del CREA informan los resultados del estudio de la validez concurrente entre el CREA C y las dimensiones de la Batería de Guilford, la cual mostró correlaciones significativas (menor a .01) de .58 para Fluidez, .77 para Flexibilidad, 68 para Originalidad y .57 para Pensamiento Divergente (Corbalán Berná, et al., 2003). El estudio de validez convergente con la prueba de figuras del TTCT, Forma A, mostró correlaciones significativas menores a .01 con el índice de creatividad (Krumm, Arán Filippetti \& Lemos, 2018). Las puntuaciones directas de cada lámina del CREA han sido tipificadas para Argentina a partir de los 6 años en adelante (Corbalán Berná et al., 2003).

\subsubsection{K-BIT, Test breve de Inteligencia de Kaufman (Kaufman \& Kaufman,} 2000)

Como medida de control, se evaluó la inteligencia. EI K-BIT consta de dos subtests: (a) vocabulario (verbal/cristalizada/conocimientos) que incluye la parte A para valorar el vocabulario expresivo y la parte B para valorar definiciones, y (b) matrices (manipulativa/fluida/procesamiento mental). Ofrece una medida de la inteligencia cristalizada (Gc) y fluida (Gf). La consistencia interna analizada con el método de las dos mitades es de .98 para el subtest Vocabulario y de .97 para el subtest Matrices. El coeficiente de estabilidad test-retest es de .94 para el subtest Vocabulario y de .86 para el subtest Matrices (Kaufman \& Kaufman, 2000).

\subsubsection{Memoria de Trabajo, WISC-IV (Wechsler, 2010)}

Permite obtener un índice compuesto de memoria de trabajo. Está constituido por dos subtest principales: Dígitos (D) que ofrece una medida de la retención verbal inmediata cuando se evalúa con dígitos directos y el mantenimiento y la manipulación de la información (memoria de trabajo) cuando se utilizan dígitos inversos. Letras y Números (LN), que consiste en la lectura por parte del examinador de una serie de números y letras desordenadas y 
el recuerdo de la serie por parte del sujeto evaluado, ordenando los números de menor a mayor y las letras por orden alfabético. EI WISC IV ha sido normalizado en Argentina. La consistencia interna promedio, calculada mediante el método de partición de dos mitades, es .85 para el subtest Letras y Números (LN), .82 para Dígitos Directos (DD) y .74 para Dígitos Inversos (DI). El coeficiente de estabilidad test-retest es .77 para LN, .76 para DD y .68 para DI (Wechsler, 2010).

\subsubsection{Test de Colores y Palabras, Stroop (Golden, 1999)}

Brinda una medida del control de interferencia y la capacidad de inhibición de una respuesta automática. La confiabilidad del test mediante el método test-retest fue de .86 para la lámina palabra, .82 para la lámina color y .73 para la lámina palabra-color (Golden, 1975).

\subsubsection{Test de Clasificación de Tarjetas de Wisconsin versión computarizada (WCST) (Heaton, Chelune, Talley, Kay, \& Curtiss, 1997)}

Brinda una medida de la función ejecutiva, particularmente de la flexibilidad cognitiva y la capacidad de categorización. Los coeficientes de estabilidad oscilaron entre .39 y .72 (Heaton et al., 1997).

\subsubsection{Fluidez Verbal Semántica (FVS) (frutas y animales) y Fonológica}

(FVF) (letras F, A, y S)

La tarea consiste en solicitar al sujeto que diga todas las palabras que recuerde, que pertenezcan a una determinada categoría (FVS) o que comiencen con una letra particular (FVF), durante el transcurso de 60 segundos. Las tareas de FV tienen normas para niños de habla hispana (Arán Filippetti \& Allegri, 2011) y son ampliamente utilizadas como medida de la FE en niños y adolescentes de diferentes países (Arán Filippetti, 2013; Friesen, Luo, Luk \& Bialystok, 2015).

\subsubsection{Five-Point Test (Regard, Strauss, \& Knapp, 1982)}

Permite obtener una medida de la fluidez no verbal o visual, definida como la capacidad del sujeto para generar respuestas novedosas. La tarea requiere de flexibilidad mental. El coeficiente de estabilidad test-retest para el número de diseños únicos fue de .77 (Tucha, Aschenbrenner, Koerts \& Lange, 2012). La actividad consiste en una hoja de papel con $\mathbf{4 0}$ matrices de puntos dispuestas en ocho filas y cinco columnas. Las matrices son idénticas a la disposición de cinco puntos en los dados. Se solicita a los sujetos que produzcan tantas figuras o diseños diferentes como sea posible en tres minutos conectando los puntos dentro de cada rectángulo. También se le informa que solo deben 
usarse líneas rectas, que todas las líneas deben conectar puntos, que no se deben repetir las figuras y que solo deben usarse líneas simples. El sujeto puede hacer diseños simples o complejos usando algunos o todos los puntos. Se da solamente una advertencia sobre la primera violación de cada una de estas reglas (Strauss, Sherman \& Spreen, 1998).

\subsubsection{Laberintos de Porteus (Porteus, 2006)}

Esta prueba permite valorar la capacidad de planificación. Consta de doce laberintos de creciente complejidad, en cada uno, el participante debe trazar el camino desde un punto de partida hasta una salida y debe evitar levantar el lápiz evitando los callejones sin salida, no estando permitido el retroceso.

\subsection{Procedimientos de recolección de datos}

Para recolectar los datos, se solicitó, en primer lugar, una entrevista con los directivos de las diferentes escuelas para explicarles las características de la investigación. Luego, se envió una nota a los padres o tutores legales de los niños explicando las características de la investigación y cómo se realizaría la evaluación. En la nota se especificó que la participación de los niños era voluntaria y anónima. Finalmente, se obtuvo el consentimiento escrito de todos los padres o tutores legales antes de comenzar la evaluación. El estudio estuvo avalado éticamente por la Universidad Adventista del Plata (Resolución 06.06/2016) y el Centro Interdisciplinario en Psicología Experimental, Unidad Ejecutora del Consejo Nacional de Investigaciones Científicas y Técnicas (CONICET), Argentina.

La evaluación se realizó en forma colectiva e individual, según las características de las pruebas, en varios encuentros con los niños. Con respecto a las pruebas que evalúan la creatividad, se siguieron las consignas de cada prueba, especificando la importancia de dar respuestas originales, fuera de lo común, diferentes y creativas.

\subsection{Análisis de datos}

Se emplearon estadísticos descriptivos: media y desviación estándar de cada tarea cognitiva empleada. Para analizar el perfil de FE según la creatividad desde el TTCT y desde el CREA se emplearon Análisis Multivariado de Covarianza (MANCOVAs), para esto se crearon cuatro grupos ( $25 \%$ ) por medio de tres puntos de corte, se suprimieron los valores medios, para maximizar la variancia primaria. Todos los análisis fueron realizados con la versión 22.0 para Windows del paquete estadístico SPSS. 


\section{RESULTADOS}

A continuación, se exponen los resultados de acuerdo con los objetivos planteados.

\subsection{Perfil de FE según la creatividad en dibujos alta y baja}

Los resultados indican que existen diferencias significativas en las FE según la creatividad medida por la prueba de dibujos $(F$ de Hotelling $(7,90)=3.404$; $p=.003, \eta^{2}$ parcial $=.21$ ). Los análisis univariados mostraron diferencias según creatividad alta y baja en (1) Índice de memoria de trabajo $(F(1,96)=$ 8.947, $p=.004, \eta^{2}$ parcial = .06); (2) Flexibilidad cognitiva espontánea: Fluidez verbal semántica $\left(F(1,96)=7.447, p=.008, \eta^{2}\right.$ parcial $\left.=.07\right)$; Fluidez verbal fonológica $\left(F(1,96)=8.998, p=.003, \eta^{2}\right.$ parcial $\left.=.09\right)$, Fluidez no verbal $(F(1$, $96)=8.323, p=.005, \eta^{2}$ parcial $\left.=.08\right)$, e $(3)$ Inhibición $(F(1,96)=14.824, p$ $<.001, \eta^{2}$ parcial $=.13$ ). No se hallaron diferencias significativas en Flexibilidad cognitiva reactiva $\left(F(1,96)=.142, p=.707, \eta^{2}\right.$ parcial $\left.=.00\right)$ y en Planificación $\left(F(1,96)=.009, p=.923, \eta^{2}\right.$ parcial $\left.=.00\right)$. En la Tabla 1 se pueden observar las medias y desvíos estándar de cada tarea de FE según la creatividad alta y baja, los datos muestran que los niños con alta creatividad puntúan más alto en memoria de trabajo, fluidez cognitiva espontánea e inhibición.

\subsection{Perfil de FE según la creatividad verbal alta y baja}

Con relación al perfil de FE según la creatividad verbal alta y baja, los resultados mostraron diferencias significativas en las $F E,(F$ de Hotelling $(7,98)=$ 8.831; $p<.001, \eta^{2}$ parcial = .39). Los análisis univariados mostraron diferencias según creatividad alta y baja en (1) el Índice de memoria de trabajo ( $F(1$, $104)=20.038, p<.001, \eta^{2}$ parcial = .16); (2) Flexibilidad cognitiva espontánea: Fluidez verbal semántica $\left(F(1,104)=25.612, p<.001, \eta^{2}\right.$ parcial $\left.=.20\right)$; Fluidez verbal fonológica $\left(F(1,104)=40.459, p<.001, \eta^{2}\right.$ parcial $\left.=.28\right)$ y Fluidez no verbal $\left(F(1,104)=31.597, p<.001, \eta^{2}\right.$ parcial $\left.=.23\right)$, e $(3)$ Inhibición $(F(1,104)$ $=19.171, p=.001, \eta^{2}$ parcial $\left.=.16\right)$. No se hallaron diferencias significativas en el dominio Flexibilidad cognitiva reactiva $\left(F(1,104)=.010, p=.921, \eta^{2}\right.$ parcial $=.00)$ y en planificación $\left(F(1,104)=2.327, p=.130, \eta^{2}\right.$ parcial $\left.=02\right)$. En la Tabla 1 se pueden observar las medias y desvíos estándar de cada tarea de FE según la creatividad verbal alta y baja. Nuevamente los datos muestran que los niños con alta creatividad puntúan más alto en memoria de trabajo, fluidez cognitiva espontánea e inhibición. 
Tabla 1.

Medias y desvíos de las FE según la creatividad baja y alta

\begin{tabular}{|c|c|c|c|c|c|c|}
\hline & \multirow{3}{*}{ INDICADORES } & \multicolumn{5}{|c|}{ PRUEBA DE FIGURAS DEL TTCT - FORMA A } \\
\hline & & \multicolumn{2}{|c|}{ BAJA } & \multicolumn{2}{|c|}{ ALTA } & \multirow[b]{2}{*}{$\mathbf{P}$} \\
\hline & & M & DE & M & DE & \\
\hline \multirow{11}{*}{ Funciones Ejecutivas } & IMT - WISC IV & 27.56 & 5.69 & 32.16 & 4.76 & .004 \\
\hline & FE: FVS & 22.46 & 6.39 & 28.04 & 6.50 & .008 \\
\hline & $F E: F V F$ & 15.86 & 7.07 & 23.51 & 8.92 & .003 \\
\hline & & & & & & \\
\hline & FnoV: FPT & 18.60 & 7.75 & 25.88 & 9.65 & .005 \\
\hline & Inhibición PC & 23.64 & 7.56 & 31.20 & 6.94 & $<.001$ \\
\hline & FR-NCC & 3.06 & 1.83 & 3.84 & 1.56 & .707 \\
\hline & Plan-TL & 11.83 & 2.17 & 12.19 & 1.86 & .923 \\
\hline & & \multicolumn{5}{|c|}{ CREA C } \\
\hline & & \multicolumn{2}{|c|}{ BAJA } & \multicolumn{2}{|c|}{ ALTA } & \\
\hline & & $M$ & $\mathrm{DE}$ & $M$ & DE & $\mathbf{P}$ \\
\hline \multirow{8}{*}{ Funciones Ejecutivas } & IMT-WISC IV & 28.65 & 5.39 & 33.82 & 4.18 & $<.001$ \\
\hline & FE: FVS & 22.36 & 4.99 & 29.43 & 5.89 & $<.001$ \\
\hline & FE: FVF & 15.40 & 6.19 & 26.22 & 7.41 & $<.001$ \\
\hline & & & & & & \\
\hline & FnoV: FPT & 17.16 & 7.53 & 27.78 & 7.77 & $<.001$ \\
\hline & Inhibición PC & 25.19 & 6.83 & 32.88 & 8.23 & $<.001$ \\
\hline & FR-NCC & 3.45 & 1.84 & 4.04 & 1.63 & .921 \\
\hline & Plan-TL & 11.45 & 2.12 & 12.29 & 1.87 & .130 \\
\hline
\end{tabular}

Notas. IMT-WISC IV = Índice memoria de trabajo WISC IV. FE: FVS = Flexibilidad espontánea: Fluidez verbal semántica. FE: FVF = Flexibilidad espontánea: Fluidez verbal fonológica. FnoV: FPT: Fluidez no verbal del Five Point Test. Inhibición PC = Palabra-color Stroop. FR-NCC= Flexibilidad reactiva - Número de categorías completas WCST. Plan-TL: Total de Laberintos correctos Porteus 


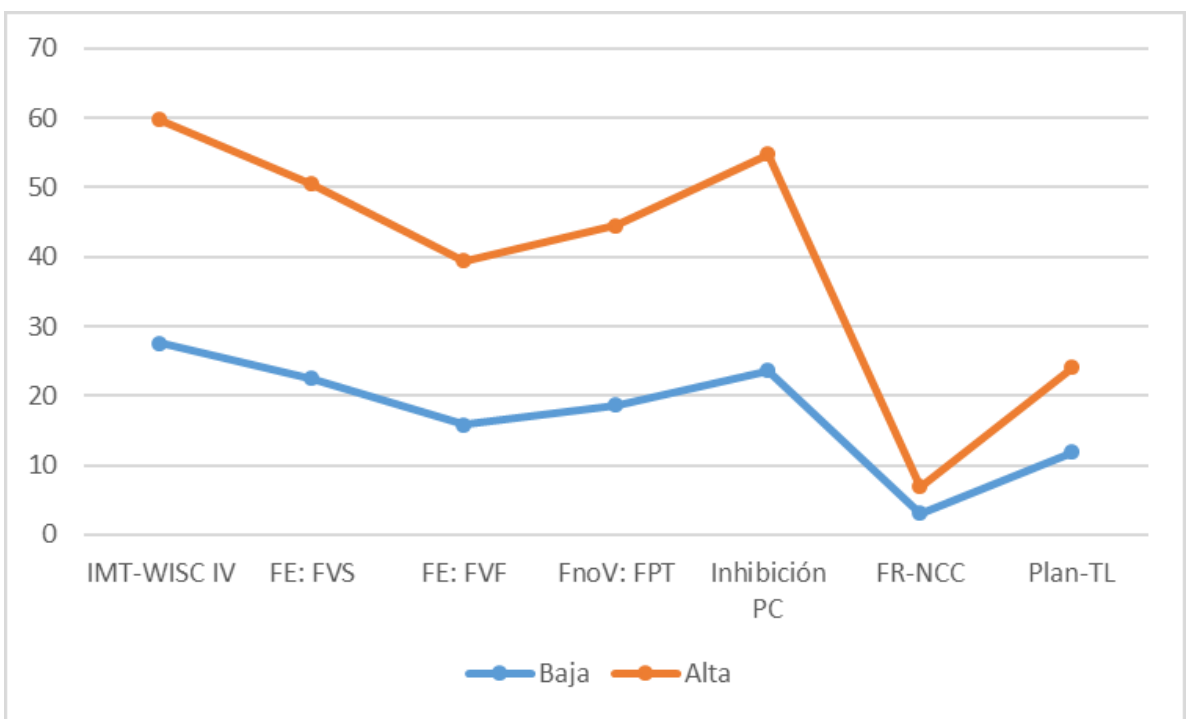

Figura 1. Medias de las FE según la creatividad baja y alta en el TTCT.

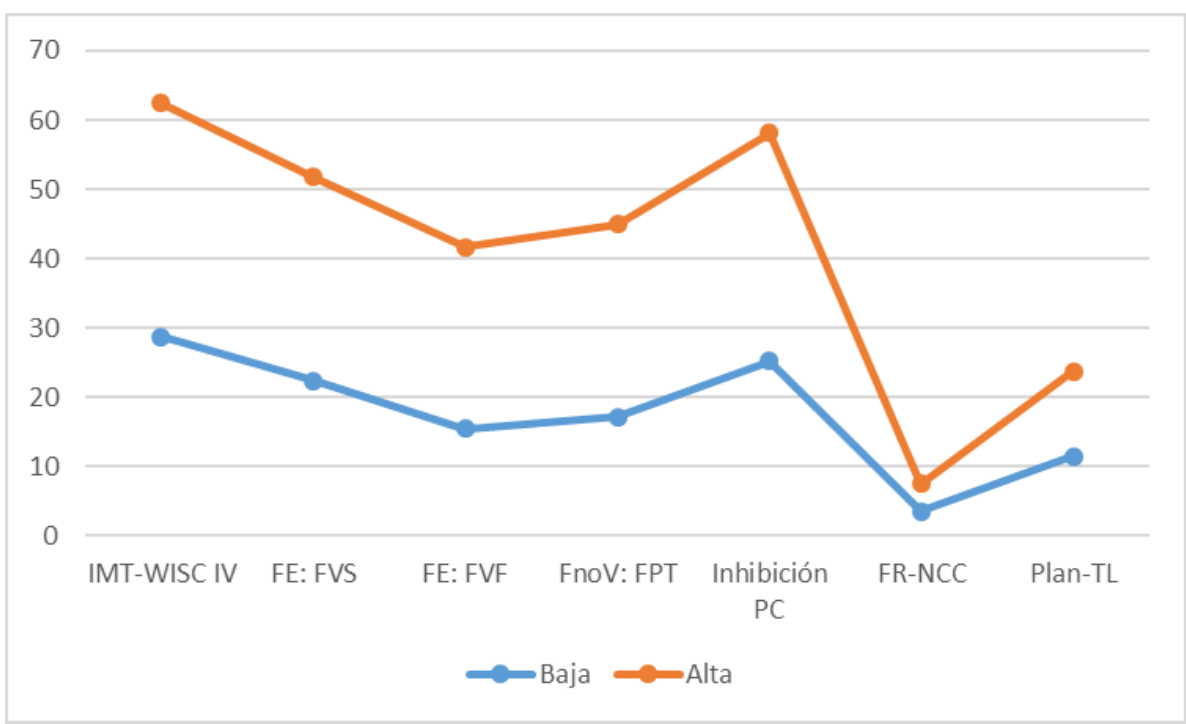

Figura 2. Medias de las FE según la creatividad baja y alta en el CREA C.

\section{DISCUSIÓN}

El propósito de este trabajo fue examinar si existe un perfil de FE característico en niños con alta y baja creatividad valorada a través de dos medidas de diferente naturaleza, una de tipo verbal y la otra a través de dibujos. En forma general, se puede observar en niños con alta creatividad un perfil caracterizado por mayor capacidad de memoria de trabajo, inhibición y flexibilidad cognitiva espontánea. Según los perfiles encontrados, pareciera que los procesos ejecutivos eficaces pueden apoyar la recuperación efectiva del 
conocimiento y así ayudar a inhibir las respuestas predominantes, y acceder a conceptos e ideas remotas y no relacionadas para combinarlas en la formación de ideas creativas (Benedek, Könen et al., 2012).

Los hallazgos respecto del papel de la memoria de trabajo en la creatividad son consistentes con lo planteado por Benedek et al. (2014), cuando menciona que las ideas creativas se originan de la asociación exitosa de conceptos previamente no relacionados tomados de la memoria (Koestler, 1964; Mednick, 1962). En efecto, la generación de respuestas en tareas de dibujo o tareas verbales escritas, con ciertos criterios, requiere de la búsqueda controlada y de la recuperación selectiva en la memoria, así como la capacidad de cambio para no repetir una idea. En este contexto, la memoria de trabajo estaría involucrada con la identificación y el mantenimiento de señales relevantes que ayudan a delimitar la búsqueda (Unsworth \& Engle, 2007). Pareciera que una mayor capacidad de memoria de trabajo facilitaría la búsqueda más eficaz, lo que conduciría a una mayor probabilidad de recuperar ideas relevantes que califican como creativas (Wiley \& Jarosz, 2012).

En relación al componente inhibición, los hallazgos están en línea con estudios previos que indican que, cuando es valorado desde el paradigma Stroop, este se relaciona en forma positiva con la creatividad (Golden, 1975; Groborz \& Necka, 2003). La inhibición refleja la capacidad de suprimir la interferencia mediante conceptos cercanos y, por lo tanto, facilitaría la activación de conceptos lejanos. En forma específica, la inhibición de la respuesta prepotente facilitaría el pensamiento creativo suprimiendo la interferencia de las tendencias de respuesta dominantes (Benedek, Franz et al., 2012; Gilhooly et al., 2007), mientras que la memoria de trabajo permitiría la búsqueda controlada y la manipulación de un gran número de conceptos. Estas dos habilidades ejecutivas, por lo tanto, pueden actuar de manera conjunta fructíferamente en la generación del pensamiento creativo (Benedek et al., 2014).

En lo que respecta al componente ejecutivo flexibilidad cognitiva, los resultados muestran que la creatividad requiere flexibilidad de pensamiento. Específicamente, se observó que la flexibilidad cognitiva espontánea caracteriza los dos perfiles estudiados, destacándose especialmente en el perfil de FE de los niños con mayor creatividad verbal. Estudios recientes han mostrado que la flexibilidad cognitiva espontánea es un predictor significativo de la creatividad evaluada desde el pensamiento divergente en los niños, siendo el mecanismo ejecutivo central que subyace entre la inteligencia cristalizada y 
la creatividad (Krumm, Arán Filippetti, \& Lemos, 2018). Además, se ha encontrado que la flexibilidad cognitiva se asocia más a los componentes cuantitativos de las tareas creativas (i.e., fluidez y flexibilidad), y no a los aspectos cualitativos como la originalidad (Pan \& Yu, 2018). Así, nuestros hallazgos son consistentes con lo informado por otros estudios previos (Benedek, Könen et al., 2012; Gilhooly et al., 2007) en los que la fluidez y flexibilidad asociativa correlacionan positivamente con la creatividad. Además, están en línea con el postulado que posiciona a la flexibilidad como un componente central de la creatividad (De Dreu, Baas, \& Nijstad, 2008). De esta forma, la flexibilidad cognitiva sería necesaria para pasar de un concepto dentro de una categoría a otro con el fin de producir varias ideas novedosas en el pensamiento creativo. Pensar de diferentes maneras lo mismo, podría facilitar las asociaciones remotas, produciendo así pensamientos más creativos; por ejemplo, considerar un barril como un instrumento musical y no como un contenedor (Pan \& Yu, 2018).

Un dato interesante es que, en los dos perfiles de FE, no se hallaron diferencias significativas entre grupos en la flexibilidad cognitiva reactiva. Esto podría deberse a que la creatividad se evaluó a través de dos pruebas dentro del enfoque del pensamiento divergente (i.e., CREA y TTCT) en las que, para calificar las respuestas, en el caso del CREA, solo se considera la fluidez y en el caso del TTCT, se consideran, en primer lugar, la cantidad de ideas pertinentes (fluidez) y luego los demás indicadores (i.e., abstracción de títulos, resistencia prematura al cierre, originalidad y elaboración). Por lo tanto, este tipo de pruebas impondría principalmente la demanda del componente flexibilidad cognitiva espontánea. Tampoco se hallaron diferencias significativas entre los niños con alta y baja creatividad en la capacidad de planificación. Considerando los escasos estudios que han demostrado que las habilidades de planificación cumplen un rol importante para el desempeño de tareas creativas que han sido realizados con adultos jóvenes (Marta et al., 2005; Osburn \& Mumford, 2006), se podría hipotetizar, en primer lugar, que la relación entre este componente ejecutivo y la creatividad depende de la edad muestral. Sin embargo, dado que se emplearon diferentes tareas a las utilizadas en los estudios previos y que, la tarea de planificación empleada en el presente estudio, al ofrecer una única posibilidad de resolución no requería de fluidez y originalidad, otra hipótesis alternativa es que la relación depende de las tareas empleadas debido a la demanda compartida entre las mismas. Por lo tanto, futuros estudios se beneficiarían de emplear diversas tareas de planificación, tanto de naturaleza verbal como no verbal, para examinar en 
profundidad si el vínculo entre esta FE y la creatividad depende de las tareas empeladas y la edad muestral.

En síntesis, los hallazgos del presente estudio muestran que los niños con mayor creatividad, tanto en tareas de dibujo como en tareas verbales, presentan un perfil de FE caracterizado por una mayor capacidad de memoria de trabajo, inhibición y flexibilidad cognitiva espontánea. Sin embargo, resulta interesante destacar que la proporción de variancia explicada por el eta al cuadrado parcial es mayor cuando se trata de tareas de creatividad de naturaleza verbal, que de naturaleza gráfica. Por lo tanto, parecería que la naturaleza de la tarea es un factor que podría modular la contribución de los procesos cognitivos de alto orden al potencial creativo.

\section{LIMITACIONES Y DIRECCIONES FUTURAS}

Si bien el presente estudio contribuye al campo de la neuropsicología infantil, al examinar la relación entre procesos cognitivos claves para el éxito escolar y en la vida, es necesario reconocer algunas limitaciones. En primer lugar, solo se utilizó una tarea para valorar la inhibición y la planificación, no así para la memoria de trabajo y la flexibilidad cognitiva. Futuros estudios se beneficiarían de incluir diferentes medidas tanto de naturaleza verbal como no verbal para valorar el constructo inhibición y diferentes habilidades de planificación. Por otra parte, siendo escasos los estudios en niños, los resultados deben tomarse con cautela a la espera de replicar el estudio en otro contexto cultural. Finalmente, es importante señalar que el presente estudio es de corte transversal. Considerando que el potencial creativo está en desarrollo, sería interesante realizar un estudio longitudinal y abordar su examen desde enfoques más inclusivos que consideren el contexto y la situación del estudio, así como la evaluación de los productos y procesos creativos involucrados.

En resumen, la presente investigación ofrece evidencias respecto de un perfil de FE específico en niños según su potencial creativo (alto vs bajo), orientando a futuras investigaciones para descubrir el rol de estos mecanismos cognitivos y de otros procesos cognitivos de orden superior en la explicación de las diferencias individuales en la creatividad. Este estudio tiene importantes implicancias educativas al poner el foco en la importancia de atender las particularidades de los estudiantes en edad escolar, ofreciendo una comprensión más profunda del papel de las FE en la creatividad. Si bien el contexto es importante para el desarrollo creativo, no se puede ignorar el papel de procesos cognitivos de alto orden, por lo que es importante generar estrategias y objetivos educativos que contemplen la promoción de las FE 
en pos de favorecer el potencial creativo. Siempre, sin perder de vista que el valor de la escuela no depende de su prestigio o su capacidad para enseñar en forma creativa a los estudiantes a enfrentarse a las necesidades de la vida sino, lo que es más importante, en qué grado es capaz de transmitir el disfrute de aprender durante toda la vida.

Conflictos de intereses: Las autoras informan que no existen conflictos de interés.

Agradecimientos: Las autoras agradecen a la Lic. Carolina Klos por su colaboración en la fase de evaluación y corrección de pruebas de FE, así como a las escuelas que participaron en el estudio.

Financiamiento: Este trabajo ha sido financiado por el Consejo Nacional de Investigaciones Científicas y Técnicas (CONICET) y la Facultad de Humanidades, Educación y Ciencias Sociales de la Universidad Adventista del Plata, Entre Ríos, Argentina.

\section{REFERENCIAS}

Arán Filippetti, V. \& Richaud, M.C. (2015). Do executive functions predict writtencomposition? Effects beyond age, verbal intelligence and reading comprehension. Acta Neuropsychologica, 13, 331-349. https://doi.org/10.5604/17307503.1187493

Arán Filippetti, V. \& Richaud, M. C. (2017). A structural equation modeling of executive functions, IQ and mathematical skills in primary students: Differential effects on number production, mental calculus and arithmetical problems. Child Neuropsychology, 23, 864-888. https://doi.org/10.1080/09297049.2016.1199665

Arán Filippetti, V. (2013). Structure and invariance of executive functioning tasks across socioeconomic status: evidence from spanish-speaking children. The Spanish Journal of Psychology, 16(e101), 1-15. http://dx.doi.org/10.1017/ sjp.2013.102

Arán Filippetti, V., \& Allegri, R. F. (2011). Verbal fluency in Spanish-speaking children: Analysis model according to task type, clustering, and switching strategies and performance over time. The Clinical Neuropsychologist, 25, 413-436. https://doi. org/10.1080/13854046.2011.559481

Ávila, N. C., López-Fernández, V., \& Arias-Castro, C. C. (2018). Análisis de la relación entre creatividad, atención y rendimiento escolar en niños y niñas de más de 9 años en Colombia. Psicogente, 21(39), 75-87. https://doi.org/10.17081/ psico.21.39.2823

Benedek, M., Franz, F., Heene, M., \& Neubauer, A. C. (2012). Differential effects of cognitive inhibition and intelligence on creativity. Personality and Individual Differences, 53(4), 480-485. https://doi.org/10.1016/j.paid.2012.04.014

Benedek, M., Jauk, E., Sommer, M., Arendasy, M., \& Neubauer, A. C. (2014). Intelligence, creativity, and cognitive control: the common and differential involvement of executive functions in intelligence and creativity. Intelligence, 46, 73-83. http:// dx.doi.org/10.1016/j.intell.2014.05.007 
Benedek, M., Könen, T., \& Neubauer, A. C. (2012). Associative abilities underlying creativity. Psychology of Aesthetics, Creativity, and the Arts, 6, 273-281. http:// dx.doi.org/10.1037/a0027059

Carson, S. H., Peterson, J. B., \& Higgins, D. M. (2003). Decreased latent inhibition is associated with increased creative achievement in high functioning individuals. Journal of Personality and Social Psychology, 85,499-506. http://dx.doi. org/10.1037/0022-3514.85.3.499

Cheng, L., Hu, W., Jia, X., \& Runco, M. A. (2016). The different role of cognitive inhibition in early versus late creative problem finding. Psychology of Aesthetics, Creativity, and the Arts, 10(1), 32. http://dx.doi.org/10.1037/aca0000036

Chi, M. T. H. (1997). Creativity: Shifting across ontological categories flexibly. In T. B. Ward, S. M. Smith, \& J. Vaid (Eds.), Creative thought. An investigation of conceptual structures and processes (pp.209-234). Washington, DC: American Psychological Association.

Corbalán Berná, J., Martínez Zaragoza, F., Donolo, D., Alonso Monreal, C., Tejerina Arreal, M., \& Limiñana Gras, R. M. (2003). CREA. Inteligencia creativa. Una medida cognitiva de la creatividad. España: TEA ediciones.

De Dreu, C. K. W., Baas, M., \& Nijstad, B. A. (2008). Hedonic tone and activation in the mood-creativity link: Towards a dual pathway to creativity model. Journal of Personality and Social Psychology, 94, 739-756. http://dx.doi.org/10.1037/00223514.94.5.739

De Dreu, C. K. W., Nijstad, B. A., Baas, M., Wolsink, I., \& Roskes, M. (2012). Working memory benefits creative insight, musical improvisation, and original ideation through maintained task-focused attention. Personality and Social Psychology Bulletin, 38, 656-669. https://doi.org/10.1177/0146167211435795

De Luca, C. R., Wood, S. J., Anderson, V., Buchanan, J. A., Proffitt, T. M., Mahony, K., \& Pantelis, C. (2003). Normative data from the CANTAB. I: development of executive function over the lifespan. Journal of Clinical and Experimental Neuropsychology, 25, 242-254. https://doi.org/10.1076/jcen.25.2.242.13639

Diamond, A. (2016). Why improving and assessing executive functions early in life is critical. Executive function in preschool age children: Integrating measurement, neurodevelopment and translational research. Washington, DC: American Psychological Association.

Dietrich, A. (2004). The cognitive neuroscience of creativity. Psychonomic Bulletin \& Review, 11(6), 1011-1026. https://doi.org/10.3758/BF03196731

Edl, S., Benedek, M., Papousek, I., Weiss, E. M., \& Fink, A. (2014). Creativity and the Stroop interference effect. Personality and Individual Differences, 69, 38-42. https://doi.org/10.1016/j.paid.2014.05.009

Friedman, N. P., Miyake, A., Corley, R. P., Young, S. E., De Fries, J. C., \& Hewitt, J. K. (2006). Not all executive functions are related to intelligence. Psychological Science, 17, 172-179. https://doi.org/10.1111/j.1467-9280.2006.01681.x

Friesen, D. C., Luo, L., Luk, G., \& Bialystok, E. (2015). Proficiency and control in verbal fluency performance across the lifespan for monolinguals and bilinguals. Language, cognition and neuroscience, 30, 238-250. https://doi.org/10.1080/23 273798.2014.918630

Fuster, J. M. (1997). The prefrontal cortex: Anatomy, physiology, and neuropsychology of the frontal lobe. 3rd Ed. Philadelphia: Lippincott-Raven. 
Gajda, A., Karwowski, M., \& Beghetto, R. A. (2017). Creativity and academic achievemen t: A meta-analysis. Journal of Educational Psychology, 109(2), 269. http:// dx.doi.org/10.1037/edu0000133

Gilhooly, K. J., Fioratou, E., Anthony, S. H., \& Wynn, V. (2007). Divergent thinking: Strategies and executive involvement in generating novel uses for familiar objects. British Journal of Psychology, 98, 611-625. https://doi. org/10.1111/j.2044-8295.2007.tb00467.x

Golden, C. J. (1975). The measurement of creativity by the Stroop color and word test. Journal of Personality Assessment, 39, 502-506. https://doi.org/10.1207/ s15327752jpa3905_9

Golden, C. J. (1999). Stroop, Test de Colores y Palabras. Madrid: TEA Ediciones.

Groborz, M., \& Necka, E. (2003). Creativity and cognitive control: Explorations of generation and evaluation skills. Creativity Research Journal, 15, 183-197. https:// doi.org/10.1080/10400419.2003.9651411

Heaton, R. K., Chelune, G. J., Talley, J. L., Kay, G. G., \& Curtiss, G. (1997). WCST, Test de clasificación de tarjetas de Wisconsin. Madrid: TEA Ediciones.

Heilman, K. M., Nadeau, S. E., \& Beversdorf, D. O. (2003). Creative innovation: possible brain mechanisms. Neurocase, 9, 369-379. https://doi.org/10.1076/ neur.9.5.369.16553

Heyder, K., Suchan, B., \& Daum, I. (2004). Cortico-subcortical contributions to executive control. Acta Psychologica, 115, 271-289. https://doi.org/10.1016/j. actpsy.2003.12.010

Kaufman, A. S. \& Kaufman, N. L. (2000). K-BIT. Test Breve de Inteligencia de Kaufman

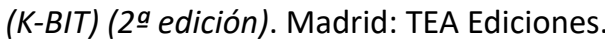

Koestler, A. (1964). The act of creation. New York: MacMillan.

Krumm, G. \& Lemos, V. (2011). Análisis de las propiedades psicométricas de la prueba de figuras del test de pensamiento creativo de Torrance (TTCT). Forma B, en la provincia de Entre Ríos, Argentina. En M.C. Richaud de Minzi y V. Lemos (Comps.), Psicología y otras ciencias del comportamiento. Compendio de investigaciones actuales (pp.731-748). Buenos Aires: Editorial Universidad Adventista del Plata.

Krumm, G., Arán Filippetti, V., \& Lemos, V. (2018). Correlates between Two Measures to Assess Creativity in Argentine Children: CREA and TTCT. In J. Penagos-Corzo \& M. A. Padilla Vargas (Eds.). Challenges in Creativity \& Psychology for the XXI century. (pp.45-60). México: Universidad de la América Puebla, Universidad de Guadalajara.

Krumm, G., Arán Filippetti, V., Lemos, V., Aranguren, M., Vargas Rubilar, J. (2013). Invariancia de Medidas de la prueba de figuras del Test de Pensamiento Creativo de Torrance según la edad: Un estudio en niños y adolescentes de habla hispana. Cuadernos de Neuropsicología, 2, 29-49. https://dialnet.unirioja.es/servlet/ articulo?codigo $=4723723$

Krumm, G., Filippetti, V. A., Lemos, V., Koval, J., \& Balabanian, C. (2016). Construct validity and factorial invariance across sex of the Torrance Test of Creative Thinking-Figural Form A in Spanish-speaking children. Thinking Skills and Creativity, 22, 180-189. https://doi.org/10.1016/j.tsc.2016.10.003

Lee, C. S., \& Therriault, D. J. (2013). The cognitive underpinnings of creative thought: A latent variable analysis exploring the roles of intelligence and working memory 
in three creative thinking processes. Intelligence, 41(5), 306-320. https://doi. org/10.1016/j.intell.2013.04.008

Marta, S., Leritz, L. E., \& Mumford, M. D. (2005). Leadership skills and group performance: Situational demands, behavioral requirements, and planning. Leadership Quarterly, 16, 81-103. https://doi.org/10.1016/j.leaqua.2004.04.004

Martindale, C. (1999). Biological bases of creativity. In R. J. Sternberg (Ed.), Handbook of Creativity (pp. 137-152). Cambridge, U.K.: Cambridge University Press.

Mednick, S. A. (1962). The associative basis of the creative process. Psychological Review, 69, 220-232. http://dx.doi.org/10.1037/h0048850

Miyake, A., Friedman, N. P., Emerson, M. J., Witzki, A. H., Howerter, A., \& Wager, T. D. (2000). The Unity and Diversity of Executive Functions and Their Contributions to Complex "Frontal Lobe" Tasks: a Latent Variable Analysis. Cognitive Psychology, 41, 49-100. https://doi.org/10.1006/cogp.1999.0734

Mourgues, C., Tan, M., Hein, S., Elliott, J. G., \& Grigorenko, E. L. (2016). Using creativity to predict future academic performance: An application of Aurora's five subtests for creativity. Learning and Individual Differences, 51, 378-386. https:// doi.org/10.1016/j.lindif.2016.02.001

Nusbaum, E. C., \& Silvia, P. J. (2011). Are intelligence and creativity really so different? Fluid intelligence, executive processes, and strategy use in divergent thinking. Intelligence, 39, 36-45. https://doi.org/10.1016/j.intell.2010.11.002

Oberauer, K., Süß, H. M., Wilhelm, O., \& Wittmann, W. (2008). Which working memory functions predict intelligence? Intelligence, 36, 641-652. https://doi. org/10.1016/j.intell.2008.01.007

Osburn, H. K., \& Mumford, M. D. (2006). Creativity and planning: Training interventions to develop creative problem-solving skills. Creativity Research Journal, 18(2), 173-190. https://doi.org/10.1207/s15326934crj1802_4

Pan, X., \& Yu, H. (2018). Different effects of cognitive shifting and intelligence on creativity. The Journal of Creative Behavior, 52, 212-225. https://doi.org/10.1002/ jocb.144

Porteus, S. D. (2006). Laberintos de Porteus (4a edición revisada). Madrid: TEA Ediciones.

Regard, M., Strauss, E., \& Knapp, P. (1982). Children's production on verbal and non-verbal fluency tasks. Perceptual and Motor Skills, 55, 839-844. https://doi. org/10.2466/pms.1982.55.3.839

Strauss, E., Sherman, E. \& Spreen, O. (1998). A compendium of neuropsychological tests: Administration, norms, and commentary. 3nd ed. Nueva York: Oxford University Press.

Sub, H. M., Oberauer, K., Wittmann, W. W., Wilhelm, O., \& Schulze, R. (2002). Workingmemory capacity explains reasoning ability-and a little bit more. Intelligence, 30, 261-288. https://doi.org/10.1016/S0160-2896(01)00100-3

Thompson-Schill, S. L., Ramscar, M., \& Chrysikou, E. G. (2009). Cognition without control: When a little frontal lobe goes a long way. Current Directions in Psychological Science, 18(5), 259-263. https://doi.org/10.1111/j.1467-8721.2009.01648.x

Torrance, P., Ball, O., \& Safter, H. T. (1992). Torrance Test of Creative Thinking. Streamlined Scoring Guide Figural A and B. Bensenville, Illinois: Scholastic Testing Service. 
Tucha, L., Aschenbrenner, S., Koerts, J., \& Lange, K. W. (2012). The Five-Point Test: Reliability, Validity and Normative Data for Children and Adults. PLOS ONE 7(9):e46080. https://doi.org/10.1371/journal.pone.0046080

Unsworth, N., \& Engle, R. W. (2007). The nature of individual differences in working memory capacity: Active maintenance in primary memory and controlled search from secondary memory. Psychological Review, 114, 104-132. http://dx.doi.org/1 0.1037/0033-295X.114.1.104

Wechsler, D. (2010). WISC IV, Escala de Inteligencia para niños de Wechsler - IV. Adaptación Argentina. Buenos Aires: Paidós.

Wiley, J., \& Jarosz, A. F. (2012). Working memory capacity, attentional focus, and problem solving. Current Directions in Psychological Science, 21, 258-262. https:// doi.org/10.1177/0963721412447622

Zabelina, D. L., Robinson, M. D., Council, J. R., \& Bresin, K. (2012). Patterning and nonpatterning in creative cognition: Insights from performance in a random number generation task. Psychology of Aesthetics, Creativity, and the Arts, 6, 137-145. http://dx.doi.org/10.1037/a0025452

Esta obra está bajo: Creative commons attribution 4.0 international license. El beneficiario de la licencia tiene el derecho de copiar, distribuir, exhibir y representar la obra y hacer obras derivadas siempre y cuando reconozca y cite la obra de la forma especificada por el autor o el licenciante.

\section{(cc) $\mathbf{B Y}$}

